\title{
Design and Implementation of PCM/FM Telemetry Systems for Aircraft and Missile Testing During Flight Test Procedure
}

\author{
Z. Filipovic ${ }^{*}$, G. Dikic ${ }^{\dagger}$
}

\begin{abstract}
This paper describes the overall system design and performance characteristics of a complete PCM/FM telemetry system for aircraft and missile testing during flight test procedure. The system encompasses subsystems for airborne data acquisition and flight line check-out, a compatible ground telemetry system. The airborne part includes a variety of component for measurement all relevant aircraft parameters. The ground telemetry system receive signal from flying object, performs real time data processing, test control and a data processing system for post flight analysis. The system represents a fully integrated approach to flight test systems which addresses the end-to-end requirements from airborne data acquisition and real time flight monitoring through aircraft performance and stability/control analysis.
\end{abstract}

Keywords: PCM/FM telemetry system, data acquisition, real time acquisition, airborne subsystem, ground station, post flight analyses

\section{Introduction}

Flight testing is not a new activity but, by definition, is as old as man's attempts to fly. Although the technology of current military aircraft is far removed from that of the first primitive gliders, the objective of flight testing remains much the same - to prove that the man/machine combination can achieve the desired "performance.

There are typically two categories of flight test programs - commercial and military. Commercial flight testing is conducted to certify that the aircraft meets all applicable safety and performance requirements of the government certifying agency. [1],[2].

Military programs differ from commercial in that the government contracts with the aircraft manufacturer to design and build an aircraft to meet specific mission capabilities. Often military test pilots and engineers are integrated as part of the manufacturer's flight test team, even before first flight. The final phase of the military aircraft flight test is perform in the Flight Test Centre by a government-only test team with the dictate to certify that the aircraft is suitable and effective to carry out the intended mission.

Flight Testing is highly expensive and potentially very risky. Unforeseen problems can lead to damage to aircraft and loss of life, both of aircrew and people on the ground. For these reasons modern flight testing is probably one of the most safety conscious professions today. Flight Testing can be divided into 3 sections, planning, execution and analysis and reporting. The purpose of a Flight Test Instrumentation System (FTIS) is to acquire data about the operation of the test vehicle and provide the data for processing during post flight analyses. FTIS can be classified in two areas: airborne and ground.

\footnotetext{
*Institute Gosa, Belgrade, Serbia, filipovicz@open.telekom.rs

${ }^{\dagger}$ Military Academy, Belgrade, Serbia
} 
The FTIS becomes more sophisticated it has become increasingly desirable to have a high quality, high speed telemetry (TM) link between the aircraft and the ground monitoring station. PCM/FM has been the primary modulation scheme used for such links for over 30 years. The telemetry system transmits the acquired data from one point to another usually using a wireless data link. Design and implementation of the FTIS is very important for successful evaluation of the testing prototype of aircraft. The configuration of FTIS is based on the General Plan of Testing of any new aircraft (or significant improvement to an existing aircraft) and missile. [2], [3].

\section{Description of the Flight Test Instrumentation System}

In the broadest terms, the FTIS is a product of hardware; software performs the functions of acquiring and presenting data to the user. In many cases, terms the FTIS, measurement system, and telemetry system are used synonymously, while in other arenas the terms are treated very differently. The broadest of these terms is perhaps the FTIS, which incorporates the measurement subsystem and the transmission/reception subsystem. A telemetry system is defined in the dictionary as any device for transmitting measurements of physical phenomena such as temperature and radiation to a distant recorder or observer. In practical terms, the telemetry system transmits the acquired data from one point to another usually using a wireless data link.

The typical FTIS can be divided into eight major functional sub-systems as shown in Figure 1. The transmission system includes the signal source, signal conditioning/processing, multiplexing and encoding, and transmission/recording of the signal.

The receiving system includes the receiving antenna and receiver, demodulation, recording and playback machines, demultiplexing, data processing, and display. [2], [3].

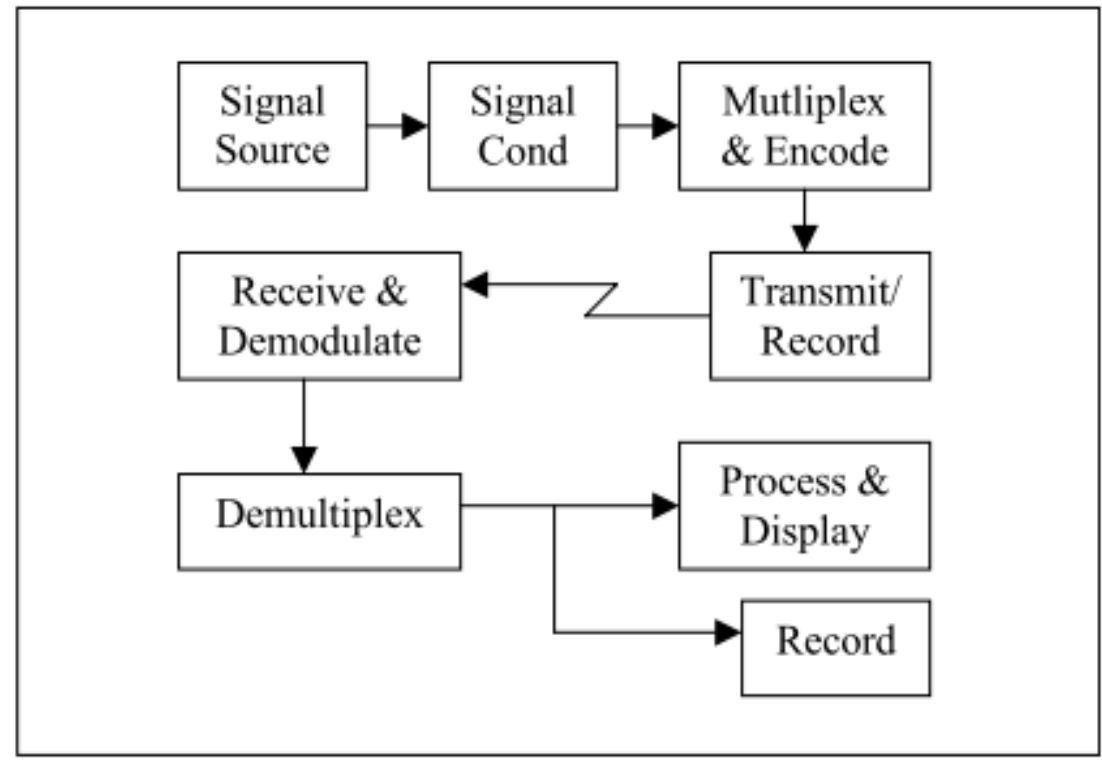

Figure 1 Sample instrumentation system.

The typical FTIS probably does not exist. Because instrumentation systems are generally unique and specialized, the instrumentation designer must tailor the design to a unique set of requirements. Consequently, the discussion of typical FTIS instrumentation system is rather difficult. However, a discussion of an instrumentation system, such as that shown in Figure 2, is instructive and serves as a good introduction to the major building blocks of modern instrumentation systems. To deliver quality data, the system definition generally begins with the design of a quality measurement system. The design and/or selection of the measurement 
devices are often the most difficult/challenging tasks in the design of an instrumentation system. The source signal may exist in a variety of forms such as electrical, mechanical, acoustical, and thermal. Since measurement systems are designed to process electrical signals, the non-electrical source information must be converted to an electrical form by using transducers. The transducer output and other forms of electrical source information are converted to more standard output forms using various types of signal conditioning equipment. Typically, more than one measurement is required and these are multiplexed prior to transmission or recording. Although many techniques are available, the most common are frequency domain and time domain multiplexing. The multiplexed (composite) signal is then transmitted to the processing station and/or recorded. [2], [3]

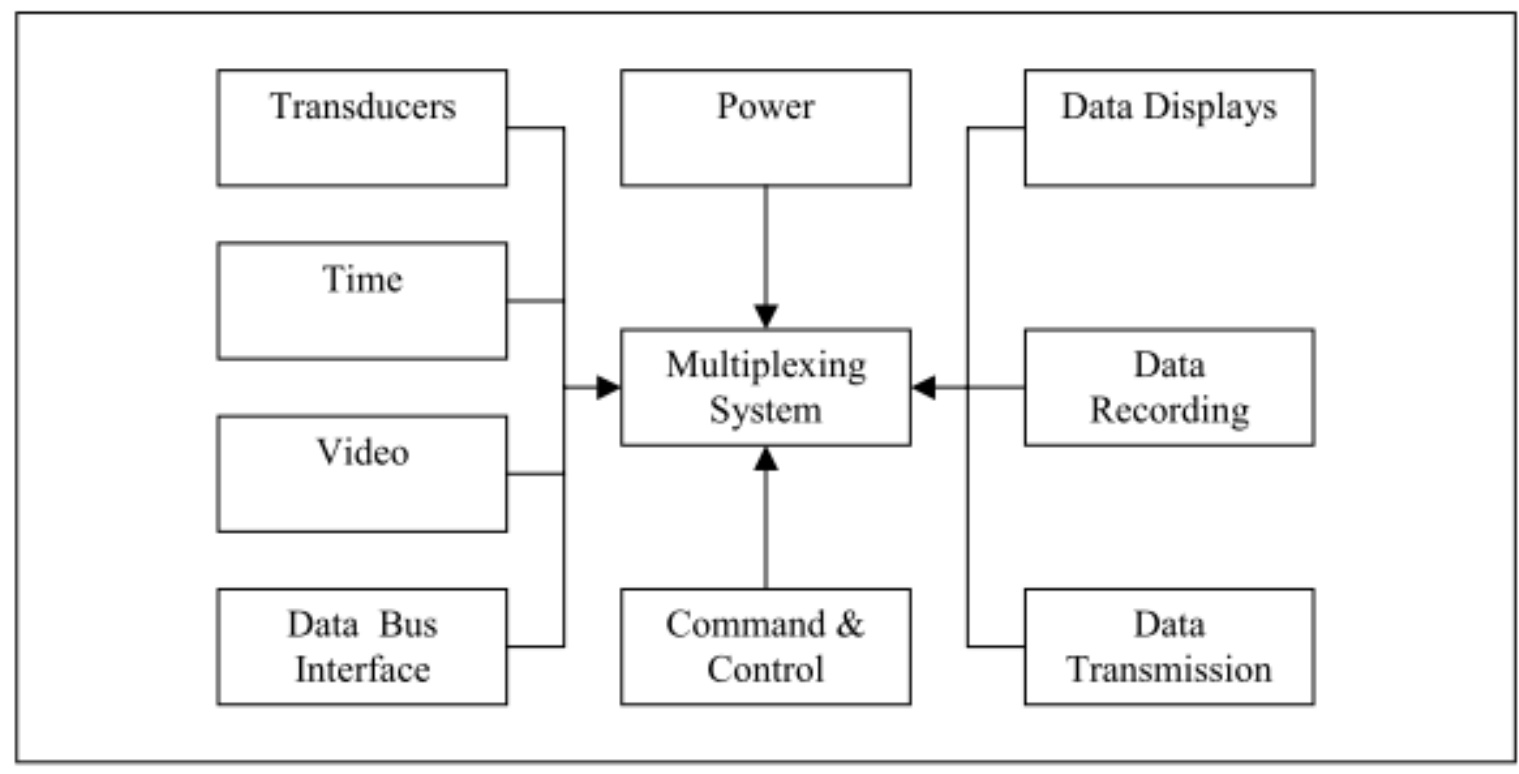

Figure 2 Typical FTIS.

The left side of Figure 2 illustrates the types and sources of information typically monitored and captured by the FTIS.In the last 20 years, the monitoring of information on data buses of aircraft and missile has become increasingly important.

In modern flight test programs of some significance, the number of digital data bus measurands is usually much bigger than the number of classical flight test measurands. The data on these buses is already in a digital format. The desired data are extracted from the data buses and are often multiplexed into the data streams with the other PCM data. As the digital words on these avionics buses are much longer than the 10-, 11-, or 12-bit words of the classical PCM words, special precautions must be taken to enable later decoding. The data bus systems used in the civil aviation community are described in ARINC429 and ARINC-629, while the military data bus standard is described in MIL-STD1553 More modern versions of the military bus standard are use, i.e., a $10 \mathrm{MHz}$ version and a fiber-optic version for even higher frequencies. [9].

The acquisition of video images is a common requirement in typical FTIS. Video signals are sometimes desirable for real-time viewing. The TM bandwidth, particularly for color, is very large. Digital encoding for encryption purposes is also possible. TM bandwidth can be made lower if lower resolution and/or frame rates are acceptable.

Transducers are the traditional source of information monitored by an instrumentation system. Some examples of measurements made using transducers are loads, temperatures, accelerations, power, and positions. The monitoring of data buses, capturing of video images, and performing measurements with transducers, is of relatively little value without knowing when the data or image was captured or when measurement was made. 
As a result, one of the most critical inputs to the instrumentation system is time. Time is used to identify when monitored events occurred (both relatively and absolutely). Without time, knowing when events occurred and correlating events would be very difficult. Also, the system designer must remember to provide power to the measurement system.

The data being monitored and measured, such as data bus interfaces, transducer outputs, and time, are sent to the multiplexing system. The multiplexing system takes the numerous data inputs and multiplexes them in frequency or, more commonly, in time. The multiplexed data is then suitable for transmission to a remote location or for recording.

In general, the data sent to the recorder will be in a different format than that sent to the transmitter. Recorders can either have analog or digital data inputs.

When transmitting data over a wireless link, two types of transmitters currently exist; these are frequency modulation (FM) transmitters and those transmitters based on quadrature modulation techniques. FM transmitters accept filtered digital data (analog data) while the quadrature modulation transmitters accept digital data.

Upon reception and/or demultiplexing, data may be sent to a data display for real time viewing in the complex mission control rooms. The common purpose of the various forms of data display is to present the data in an accurate and easily understood manner. [2], [3], [9].

\section{1 Signal Sources and Sensor Selection Criteria}

There are a lot of source for a measurement during flight test procedure of any prototype of aircraft or missile .Temperature, batter, voltage, vehicle speed, altitude, heart rate, wind speed, and rudder position are all examples of sources of measurement signals. For most of these sources, a sensor (or transducer as they are commonly called) must be used to convert physical energy into electrical energy. The electrical signals, whether it is current, voltage, or frequency are proportional to the physical energy they monitor. Serial information and MILSTD-1553 data bus information can also be considered signal sources.

Selecting the right sensors from among the wide variety of sensors available on the market today depends on the particular need for the parameter being measured. Before selecting the sensor, the instrumentation engineer should address the following questions for each measurement task:

- Type. Is there a transducer that measures your physical phenomenon?

- Range. What are the maximum and minimum values expected to be measured?

- Sensitivity. Is the ability to discriminate small changes in the measured quantity sensitive enough?

- Accuracy. How close is the sensor to the actual value?

- Repeatability. What is the ability of the sensor to reproduce an output given the same input?

- Resolution. What is the smallest signal increment that can be detected?

- Response Time. How fast (a value of time or frequency) can the sensor reacts to a stimulus?

- Cost. What is the value for the amount paid while still meeting requirements?

- System Integration. Is the output compatible with inputs to your data collection system?

- Special Needs. Are there special mounting requirements, hazardous environments, or other special needs?

Initial planning should include a review all the above questions to determine which ones are applicable, and to identify the relative importance of some over others. For example, speed and accuracy usually come with a price, so knowing what is needed can help keep costs to a minimum. Special operating parameters and conditions may also dramatically increase costs, so determining how parameters are to be measured, in addition to what is to be measured, 
should be considered in the initial plan. The final determination of measurement requirements is usually a compromise of several parameters to fit within a specified budget. Knowing the exact measuring requirements is critically important to the selection of the correct sensors. [2], [3].

\section{2 Signal Conditioning}

Signal conditioning is the modification of a signal from a signal source to some final form. Modification steps can include amplification, filtering, sampling, digitization, data compression, digital formatting, modulation, correction factors, and conversion. Generally speaking, for signal conditioning is use the definition that signal conditioning is any signal conversion between the signal source and the data capturing system. An example would be adding gain and offset to a resistive temperature device (RTD) before it is time-sampled. Another example would be the capturing of a data word in a MIL-STD-1553 message stream and adding a time stamp before it is placed in a pulse code modulation (PCM) stream.

Signal conditioning can be categorized as either analog or digital. Analog signal conditioning can consist of amplification, attenuation, filtering, zero shifting, and compensation. Digital signal conditioning consists of converting a digital signal into another digital form for data capture, transmission, or recording. Digital signal conditioning can be as simple as parallel to serial conversion or as involved as MIL-STD-1553 data capture. [2], [3].

\section{3 Data Collection and Pulse Code Modulation (PCM) Formats}

Data collection is the process of gathering all the necessary information from a set of sensors (such as transducers communications buses, etc.) and putting the information into an organized format. The newly formatted information can then be used by a data analyst to evaluate the performance of a system or event being tested. The system being tested could be as large as an aircraft, or as small as a missile. There are different configurations of the FTIS available and choosing one depends upon many criteria such as accuracy, cost, data rates, test environment, and space requirements.

PCM formats, referred herein simply as formats, identify the manner in which data is organized and/or provided by an instrumentation system. Because formats may be recorded or transmitted for data analysis, the format of a data collection system must be programmed According to the data parameters list which is need to measure during flight test procedure, it is necessary to create a format in which all data is sampled at a high enough rate to prevent loss of information.

Bus data should be acquired at a rate just above the bus data rate. Analog data should be sampled at different rates depending on the type of data; for example, vibration data will generally be sampled at a higher rate than temperature data. When the PCM format has been created, it is then programmed into the data collection system for testing. [1], [9].

\subsection{Telemetring}

Telemetry is the method used for sending information from one place to another. Examples of this method are cable modems (modulator/demodulator), Ethernet, and even point-to-point serial communications. In the world of instrumentation, telemetry (TM) system deals with the transmission of test data to some receiving station. The transmission method medium can be air or wire depending on the application. Aero telemetry everything required to convert data from baseband to radio frequency (RF) and back again.

TM systems are used either in conjunction with airborne recorders to transmit portions of the entire data stream to the ground or with only ground recorders when the entire data stream is transmitted such as tests of air-to-air or air-to-ground missiles. Telemetry without on-board recording may also be necessary for cost, safety, or space reasons. 
For most military testing, encryption of any transmitted PCM data is required. This requires additional equipment both on the vehicle to randomize the PCM bit stream and on the ground to recover the original data. Availability of special encryption equipment for both the vehicle and more particularly, ground play-back, is often a problem; also, the more items or procedures that are inserted between the measurand and the FTIS, the more possibility of failure or a misunderstanding. The delay in receiving processed data from a recorder must be balanced against total data loss if an encryption failure occurs (all TM) and the ability to monitor system health, safety, and security requirements for an encrypted vehicle.

The TM actions include modulation of the baseband data (information signal), transmission, acquiring, receiving and demodulation of the signal back into its original baseband form. On the test platform side, the telemetry system includes the information signal, any required encryption or pre-modulation filtering, telemetry transmitter, power splitter, and antenna(s). On the receiving side, the telemetry system includes the reception antenna, telemetry receiver, demodulator, decryptor, and bit synchronizer (referred to as "bit sync").

Modulation is the process of attaching information to a carrier wave that involves the variation of one of the carrier-frequency characteristics. Traditional systems have used the method of Continuous Phase Frequency Shift Keying (CPFSK), more commonly referred to as PCM/FM, to attach the information to the RF carrier. Another method utilizes a variation of Quadrature Phase Shift Keying (QPSK) modulation techniques, which reduces the transmitted spectrum. The QPSK technique is mainly used in applications requiring higher bit rates. [2], [3].

Many parameters must be considered when designing or analyzing the performance of a telemetry link. These parameters include the following:

- Link margin,

- Error probability,

- Pre-modulation filtering,

- Transmitter deviation,

- Antenna location and patterns,

- Power split between antennas,

- Data rate,

- Antenna-tracking loops,

- Receiver set-up.

\section{5 Recording}

The majority of instrumentation systems include some type of recording device to analyze data after the completion of a test. Many types of recorders collect video and data information. Determining which recorder will be used is normally determined from the required testing, space availability, power requirements, cost, and supportability.

Determining the requirements for the recording device is an important step in selecting the correct type and unit for the instrumentation system. When selecting a recording device for an instrumentation system, the following issues must be taken into account: [2], [3]

- Recording time,

- Data rate,

- Number of data streams,

- Space availability,

- Environnemental conditions,

- Availability of funds,

- Playback capabilities. 


\subsection{The Ground Receiving System}

The airborne subsystem of the FTIS must also consider the requirement for and the need for compatibility with ground systems. A special item of ground instrumentation is the ground telemetry station (GTS). In this station the telemetry signal is received and processed to present data to the ground-based personnel during flight. There are two regimes of working of the GTS:

- Real time processing data,

- Post Flight processing data.

This system can be sophisticated, with specialized computers and graphical workstations giving engineering units of airborne measurement data. During the flight test received measurement data record by special Ground recorded during as a part of the GTS.

Thanks to special software which is part of the FTIS, it is possible to have complete information about tested object in real test time and during postflight analyses. [1], [2].

\section{An Example of the Design of a Typical FTIS}

The total FTIS is split into 2 parts, the airborne system and the ground station. The airborne equipment comprises the RF transmitter, multiplexer and the antenna system. The ground station consists of the receiving antenna, a demultiplexer that feeds the data to a distributor and onwards to the monitoring and recording equipment, which presents the flight data to the user.

When a new test of aircraft or missile is in the planning stages, before testing begins, it is common to have considered the scope and complexity of the test. The time schedule, test plans, range support, and system operation, must be deliberate.

The instrumentation engineer who is responsible for design the FTIS needs to know the answers to several key questions as soon as possible because early planning is the key to the successful deployment of the FTIS. Sometimes he may have to use his education, experience, and common sense to "suggest" the right solution to questions such as: [2], [3].

- What measurements (list of parameters) are desired and how often (i.e. sample rate)?

- Can vehicle power be used for instrumentation purposes?

- Where can the instrumentation are mounted?

- How much space is available?

- Is there a weight limitation?

- In what type of environment will the instrumentation and transducers be exposed?

- Will access to the test item be provided prior to test in order to take measurements, make drawings, acquire sample data, etc.?

- Are there any communications systems that will be used on the test item?

- Can an instrumentation antenna be mounted on the test item?

- Is there a real-time requirement for system control?

\subsection{Airborne Part of the FTIS}

The airborne data acquisition subsystems were required to be general purpose to satisfy the wide range of data acquisition problems normally found in modern aircraft test programs.

General requirements included:

- Acquisition and PCM output of normal analog voltage, frequencies and discrete events

- Programmable sampling plans

- Recording and telemetry transmission of PCM

- Acquisition of data from two MIL-STD-1553B busses

- Output of selected 1553 bus words in the PCM stream

- Recording of $100 \% 1553$ data from both busses 
- Color video data recording and telemetry transmission

Figure 3 illustrates the typical configuration for a system. General aircraft measurement data is acquired using an airborne part of the FTIS. This is a remotely multiplexed system which provides a maximum PCM bit rate of 3.2 Megabits per second or more with a maximum total sample rate of 200,000 samples per second. PCM formats generated may use variable word lengths. This allows analog channels to utilize word lengths of 8,10 , or 12 bits and digital channels to utilize word lengths of up to 16 bits. Each remote signal conditioner chassis may contain up to 16 analog or digital conditioner cards which may contain from 2 to 16 channels each depending on the conditioner type. Format and sample plan data are loaded into the system's EEROM memory via an RS232 port.

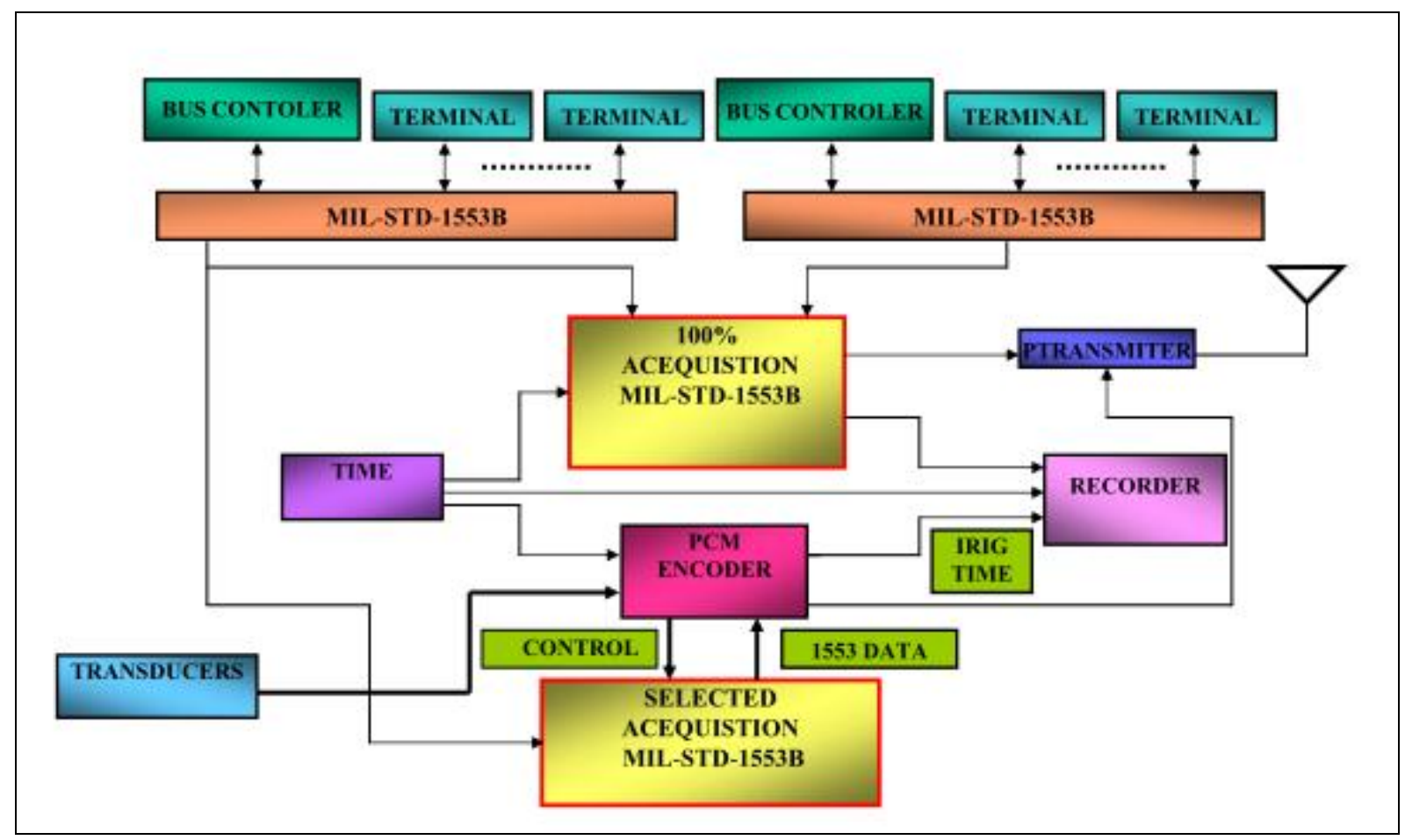

Figure 3 The configuration of airborne part of the FTIS [1]

The airborne 100\% 1553B bus data unit use for acquisition full traffic bus from multiple busses in accordance with the Inter-Range Instrumentation Group (IRIG) standard. The Airborne 1553B bus word selection unit select a number of data ( 1024 words) from redundant busses for insertion in the general PCM ( data selector card which interfaces to the PCM Encoder ).

The airborne time code generators provide serial time code for recording, and parallel time code for input to the $100 \% 1553 \mathrm{~B}$ bus unit for data time correlation. The airborne instrumentation recorders are provided for direct recording of all data. The Video cockpit color video cameras and airborne video recorders are supplied for video data acquisition.

Dual L or S-band telemetry transmitters operating on different frequencies are used for the simultaneous transmission of video and PCM telemetry data. The outputs of the transmitters are mixed in a diplexer and the resulting signal is split between two transmitting antennas normally located on the top and bottom of the aircraft. [1],[8],[9]

A wide assortment of Signal Conditioners, Bus Monitors, and other special function cards can be installed in any combination. Signal conditioning is completely programmable from excitation through PCM output. All operational parameters are set at the system, card, and frame level using easy to follow menus and familiar Windows ${ }^{\circledR}$ commands. The PCM System encoders are designed to provide maximum accuracy of test results in the most severe environments. All housings are environmentally sealed with EMI/RFI shielding and gasketing 
and meet MIL-STD-810E requirements. To minimize noise and crosstalk, all plug-in slots are individually shielded in the housing.

The Standard housing provides 16 card slots and up to 256 signal conditioned channels. MiniHousings, ideal for small enclosures and rotating platforms, provide 4 card slots with up to 32 signal conditioned channels. A combination of housings can be used in the Master-Slave configuration. The airborne part of the FTIS Data is versatile and flexible which can be easily adapt to changing test specifications and future needs. [1] [7],[8].

High-G telemetry system for tank munitions measurement of in-flight motion characteristics of a high-G launched flare-stabilized projectile is shown in Figure 4.

A $25 \mathrm{~mm}$ flare-stabilized projectile consisting of an antenna, transmitter (TM), encoder, regulator, power supply, inertial sensor suite and a removable turn-on swich. There are three axis of acceleration, two axis of angular rate, three axis of magnetic field measurement, temperature and two additional accelerometers providing a direct measurement of spin.

This system has demonstrated survivability to 25,000 G's in ground based evaluations and 20,000 G's in a spin stabilized projectile.[5]

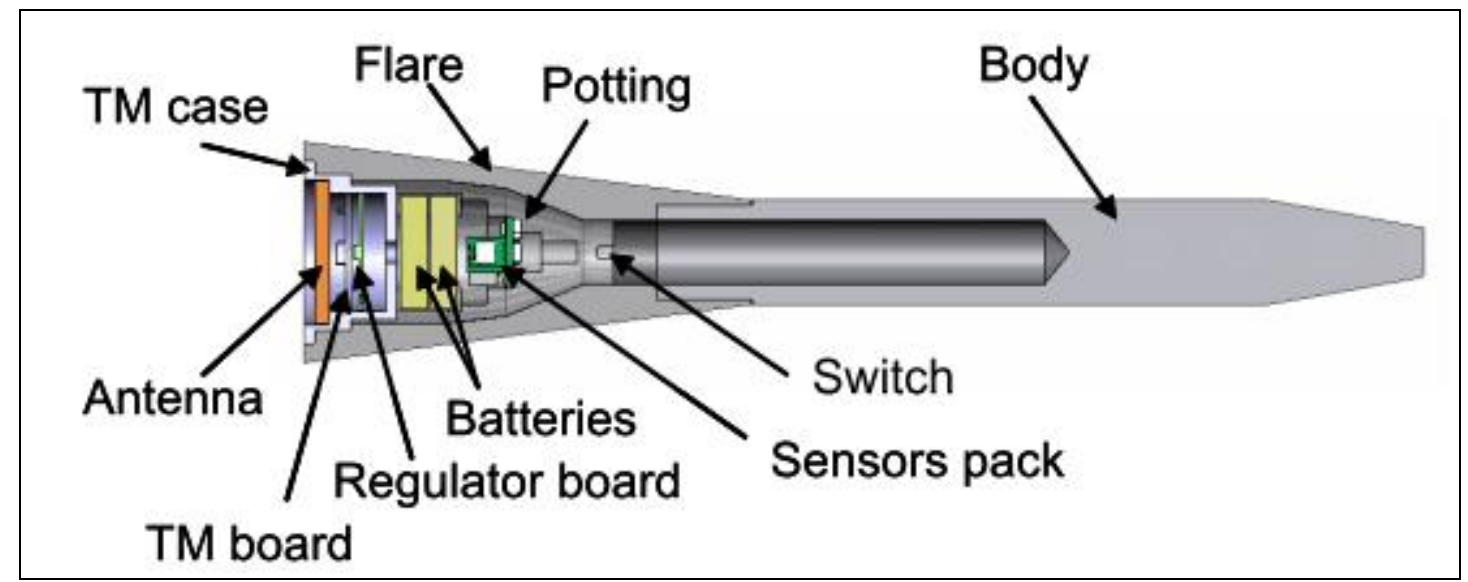

Figure 4 The Cross-section of projectile and electronic components. [5]

A practical transmission part of the FTIS for transmission measurement data to the GTS in severe environments is shown in Figure 5.

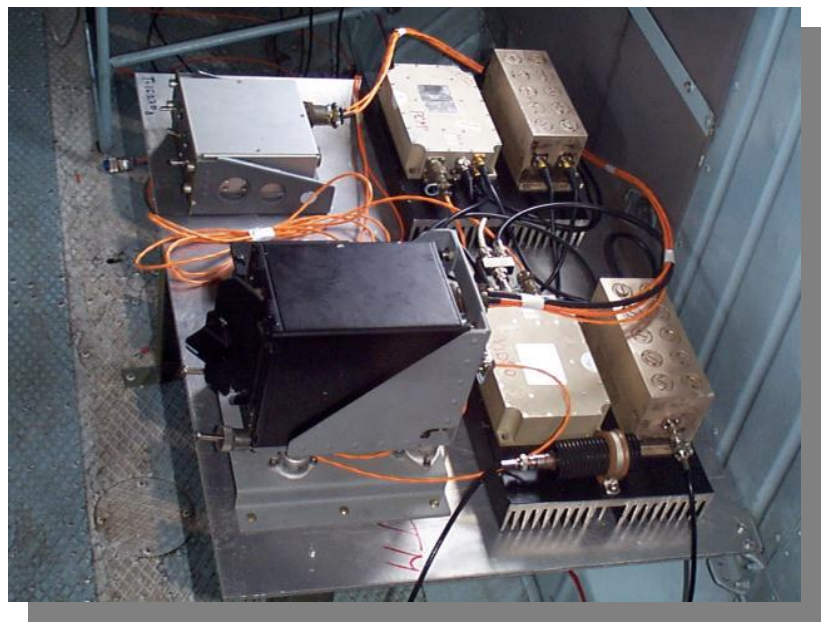

Figure 5 The configuration of airborne transmission part of the FTIS [1] 


\subsection{The Ground Telemetry Station}

The equipment and software installed in compatible ground part of the FTIS (Figure 6) supports real time flight test operations and is required to provide the following capabilities in real time:

- Dual axis tracking antenna

- PCM and video telemetry reception and recording

- PCM telemetry or instrumentation tape playback processing

- Real time workstation displays for test director

- Independent real time workstation displays for engineering personnel supporting the test

- Real time control system transfer function analysis (expandable to real time flutter analysis in the future)

- Disk data formatting with inter maneuver playback analysis

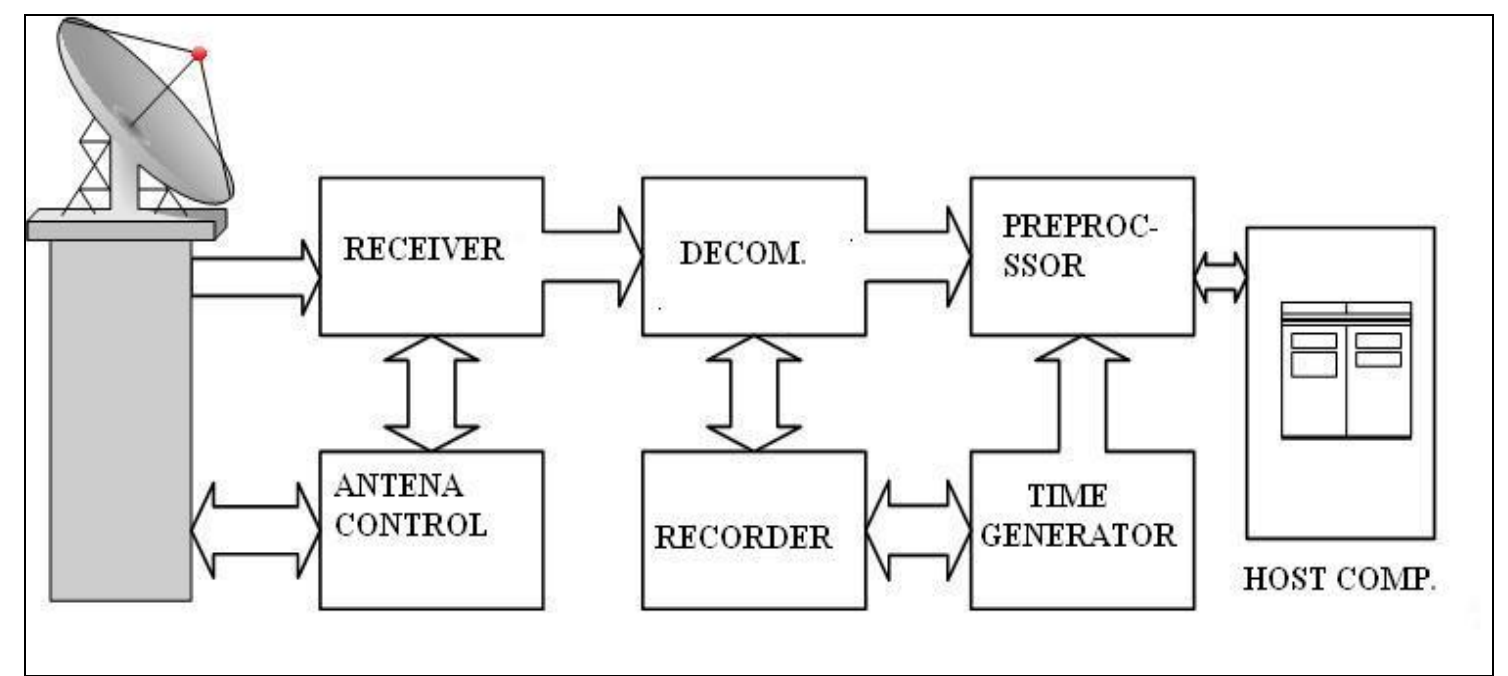

Figure 6 The Basic configuration of the GTS [1]

The L(or S)-Band dual axis tracking antenna receives the RF telemetry signal containing both the PCM and video data.

Dual receivers and a diversity combiner equipped for both pre- and post-detection combining are provided for the PCM data to improve the signal quality. A single receiver is utilized for the video data which may be both recorded and viewed on the monitor.

The instrumentation recorder/reproducers use for the recording of telemetry data during real time and playback of tapes after the flight..

The GTS is equipped with a PCM synchronization/decommutation hardware which provides a high performance input link for the telemetry data. It is equipped for the playback processing of three simultaneous streams of PCM data from instrumentation tape - one general-purpose PCM stream, and two PCM streams containing $100 \%$ of the data from each of two MIL-STD-1553B busses.

The three primary system computing resources are the preprocessor, the host processors, and the workstations.

All input data streams from both systems are input to an Telemetry Multiplex Processor. This preprocessor performs all preprocessing tasks including 1553 data decommutation, engineering units conversion, limit/events alarm checking, simple derived calculations and other commonly required telemetry functions using parallel word slice, floating point processors. 
The GTS utilizes the Host computer, for processing of different data streams and formats. This capability is made possible through configuration support software. This software allows the user to define and logically partition the input, output, and parallel processing modules of the preprocessor among multiple input stream processing tasks. Each set of modules can then be independently loaded with a format processing definition and processing can be started, stopped, or reloaded independently of what is occurring within other logical partitions. An array processor is included with the GTS for real time transfer function computation.. The array processor performs FFT and vector arithmetic operations for calculation of transfer functions

An Ethernet local area network interconnects several host processors, a number of engineering workstations for monitoring received data during flight. Any of the workstations may independently logon to either host for maximum utilization of facility resources.

The host computer is allocated the functions of database management, data acquisition control, data archiving, derived parameter calculation, data analysis and data distribution. The workstations provide the system user interface, perform data display, do hardcopy formatting of displayed data, and run a powerful graphics display editor. [1] , [5] , [6] , [7], [8]

An example of presentation of air to ground bombs trajectory in real time in the Control mission centre processing by the GTS is shown in Figure 5.

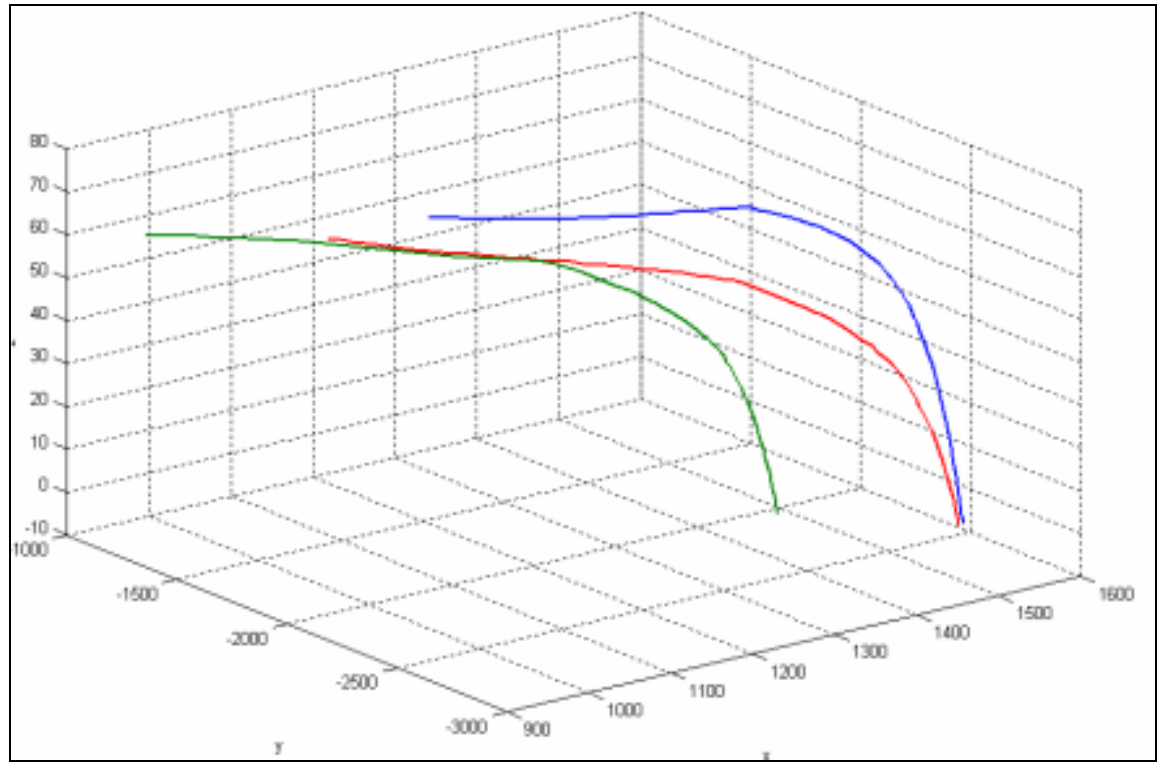

Figure 8 Real time presentation air to ground bombs trajectories [1]

\subsection{Operational Considerations}

Numerous operational aspects must be considered as part of the system design. Calibration, the determination of the over-all transfer function of each measurand from the sensor to the encoder, must be carefully thought out. System calibrations are also called "end-to-end" calibrations. They are performed after the system is installed in the test vehicle.

The calibration process is a method of reducing errors in the measuring instrument by calibration against a known standard, usually of higher accuracy than that required from the measurement. Calibration cannot reduce errors but it does allow some of them to be quantified. Calibration is also the process that relates the input to the output of a particular instrument thus defining the system characteristic. The general characteristics of the instrument eg. frequency response, damping, linearity etc should already be known beforehand when the FTIS requirements are notified and the calibration process will establish the precise relationship of the input to the output. 
The calibration process relating to the primary input parameter also usually consists of both a static and dynamic calibration and hence the complete calibration process may be categorized in three parts:

- Static calibration: To determine the relationship between the input parameter and the output of the instrument without the dynamic characteristics of the system intruding.

- Dynamic calibration: Derivation of the response characteristics of the system to dynamic inputs. (Usually presented as an amplitude ratio and a phase difference as a function of frequency).

- Environmental calibration: To include all the environmental factors that can affect the output.

A calibration source is used to stimulate the transducer. The system designer monitors the signal entering the data collection unit to ensure the calculated value matches the actual system transfer function. Since the system calibration is performed with the same measurement system (including wiring, connectors, routing, etc) as will be used during the test, many potential problems such as phasing and wiring errors can be identified corrected early in the process. On-vehicle calibrations usually do not provide for error determination of environmental effects. Checks for this in a laboratory may be needed. Occasionally special spot-check items are designed, particularly when verification item availability is limited, or handling, special hanger, etc., preclude operational access.

Removable transducers can be calibrated in a laboratory and environmental effects can usually be determined.

The final objective of calibration is to determine the characteristics of a complete chain of measurement. In summary therefore, the calibration process may be used to:

- Check that each individual component within the FTI S is functioning correctly.

- Establish the characteristics of the input and output of the complete measurement chain allowing conversion to engineering units for post-flight analysis.

- Determine the accuracy of the measurement system relative to the input.

Each of the above calibrations requires the use of a standard to measure both the input and the output and the accuracy of these standards must be chosen carefully as it will be defined by the overall accuracy of measurements required. Since the calibration error contributes to the total error it must be sufficiently small to ensure that the total error does not exceed the required value.

Pre/post-flight operations involve serious checking all relevant parameters in order to discover potential problems.

An example of necessary vibration measurement and calibrations of prototype of aircraft before beginning of the flight test procedure is shown in Figure 9.

After performing a number of flights for checking all components of the FTIS, the flight test procedure can start in order to carry out a lot of flights related to the Test Plan of the aircraft.

As results of this process is the Final report of testing including all significant data of implementation all relevant standards concerning subsystems and whole object of testing. [2], [3],[7].

\section{The Link Improvements of PCM/FM Telemetry Systems}

One of the main tasks for the Instrumentation engineer who is responsible for design of the FTIS is improving the PCM/FM link performances in the following areas:

- BER Performance

- Spectral Occupancy

- Link budget requirements

- Resynchronization time \& link availability 
- Performance with distortion (e.g. multipath fading)

Multipath interference is a common cause of signal degradation in aeronautical telemetry.

Multipath occurs when multiple copies of a transmitted signal reflect off various surfaces and recombine at the receive antenna. Having traveled different paths, these multiple copies have different phases and amplitudes. The phase differences between the paths vary as the relative

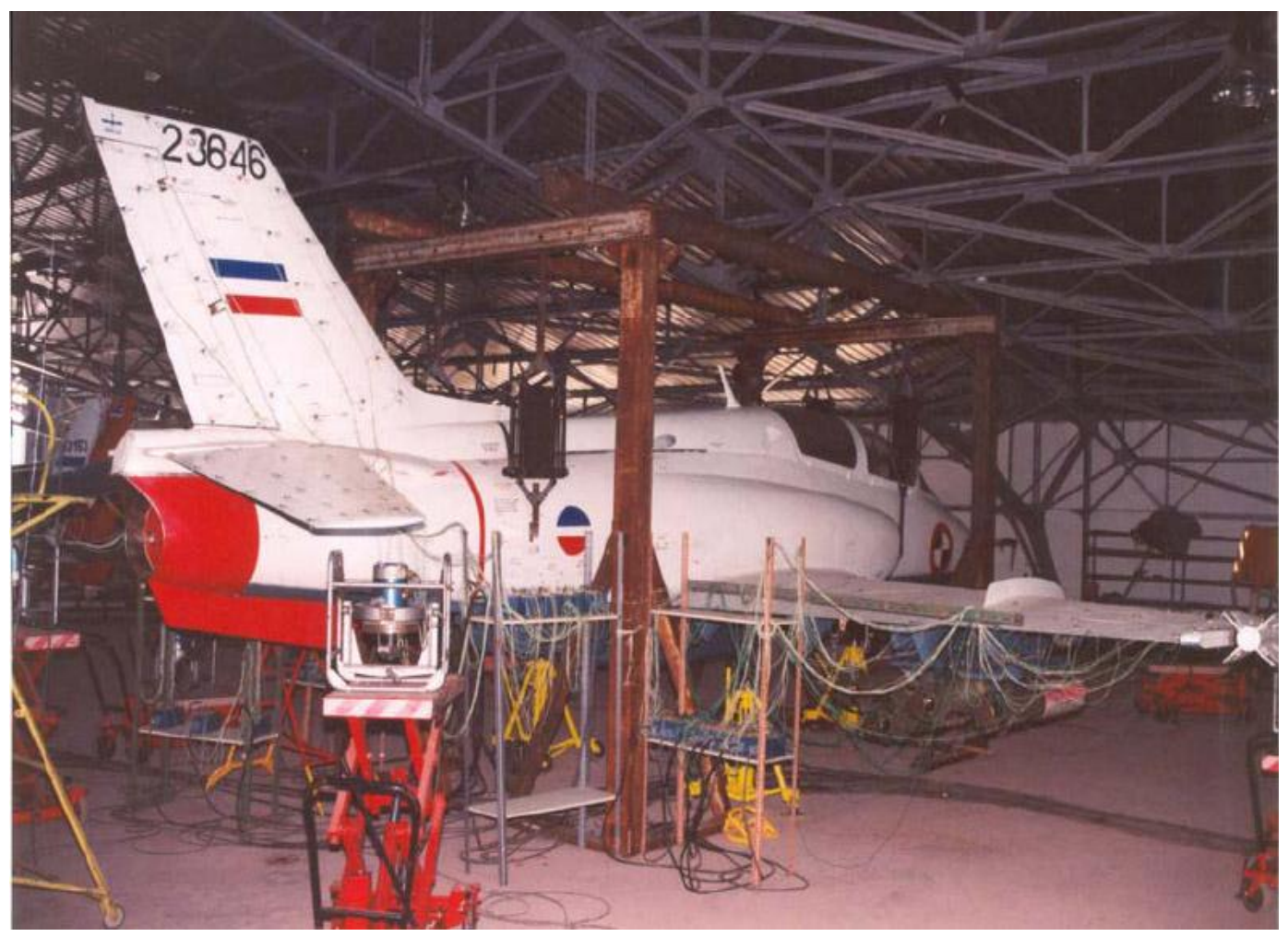

Figure 9 The ground vibration measurement and calibrations before flight test procedure[1]

positions of transmit and receive stations change with time. This results in random periods of both constructive and destructive interference. The phase of the received copies is a function of the dielectric properties of the reflecting media, the delay imposed by the differential path length, and the wavelength of the electromagnetic wave front. Since the phase of received copies is a function of frequency, multipath may cause constructive interference at one frequency and destructive interference at another. When both frequencies are within the bandwidth of the transmitted signal, part of the spectrum of the transmitted signal may be attenuated while another may be unaffected. This phenomenon is called frequency selective fading. [4] , [5]

In order to overcome this problem (increase BER Performance) during application of PCM/FM Telemetry Systems it is necessary use $t$ diversity technique and Forward Error Correction (FEC).

Many PCM/FM links include some form of transmit or receive diversity. Diversity is a method of improving link quality by transmitting or receiving two or more versions of a signal and adaptively combining or selecting the best signal so that the resultant bit error rate is lower than for any one version of the signal alone. There are a variety of schemes for separating the diversity signals including spatial diversity, frequency diversity and polarization diversity. 
In PCM/FM systems diversity combining is often performed at the IF (Intermediate Frequency) frequency based on relative noise power in the received signals - this is known as pre-detection diversity combining. Other forms attempt to weight two or more demodulated streams of data at baseband frequencies (called post-detection combining) or use metrics such as lost sync words or baseband noise level to dynamically switch between streams (called best source or best data selection).

There are a variety of FEC codes in use in wireless communications systems today, including many variants of convolutional codes, turbo codes and low density parity check DPC codes. All of these techniques rely on adding some redundancy to a stream of data so that the receiver can detect and correct a proportion of the errors that occur during transmission.

The code which is widely used in telemetry products is a half-rate convolutional code with $\mathrm{k}=7$. This code type has the advantage of using a very simple airbone PCM encoder (thus reducing airborne hardware complexity) and is a streaming code so that no framing is required. This allows resynchronization to occur rapidly after a reception outage.

A typical PCM/FM transmitter assembly consists of a PCM encoder, pre-modulation filter, FM transmitter and transmit antenna. A pre-modulation filter may be thought of as component that accepts a sequence of impulses generated from the data stream and filters these impulses for the purpose of spectral containment after modulation.

Traditional pre-modulation filters are implemented using analog components and as such are limited to a specific characteristic and data rate. However implementing an all-digital premodulation filter as provides a greater degree of integration, thus saving on space and equipment costs. This type of filter has the capability of handling multiple data rates and variable output amplitude. Moreover the digital nature of the filter allows selection of virtually any pulse-shape. The major advantage of digital filters, there is no intersymbol interference (ISI) at multiples of the symbol period from the ideal sampling point. The PCM/FM modulated RF spectra (S-band) for different tapes of digital premodulation filters (Tier-0 PCM/FM $6^{\text {th }}$-order Bessel Filter -, BRRC- Binary Root-Raised Cosine pulses and QRRC- Quaternary Root-Raised Cosine) which satisfied IRIG-106 Standard related spectral occupancy is shown in Figure 10.

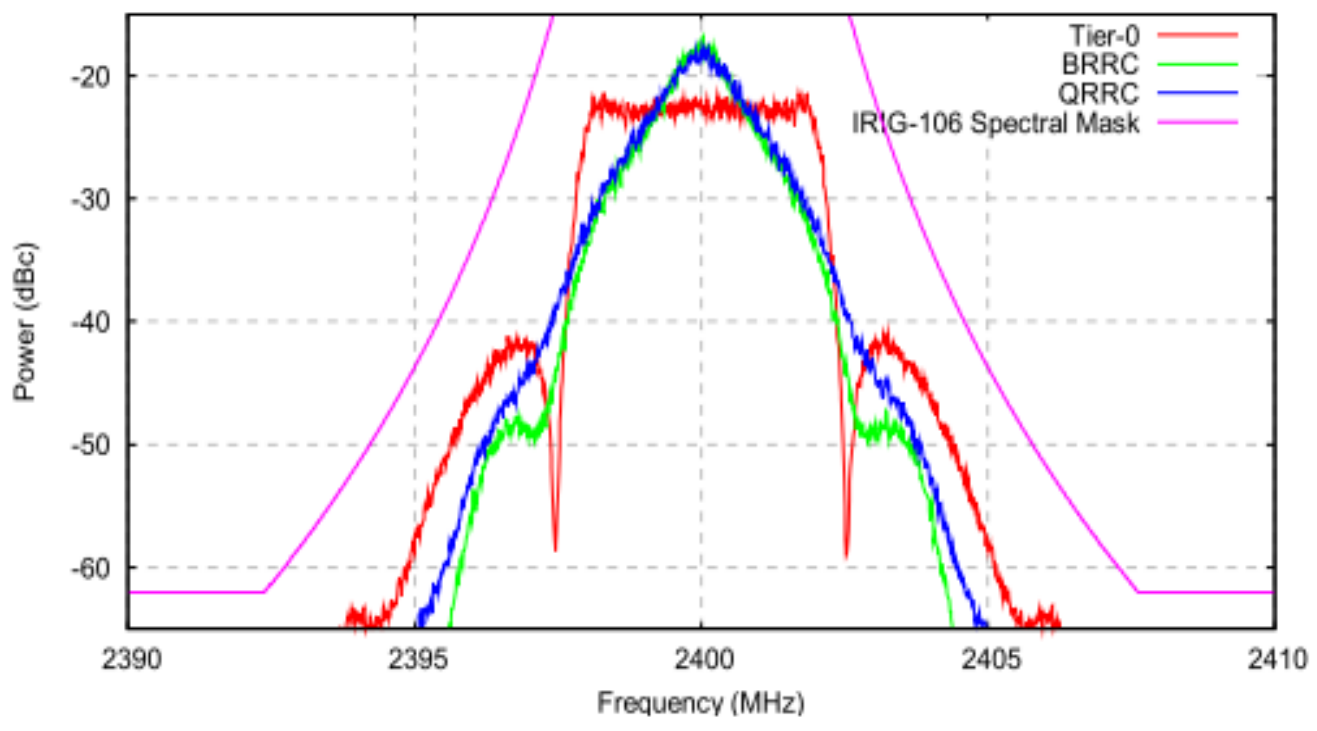

Figure 10 PCM/FM modulated RF spectra for different Pulse shapes of premodulation filters [6] 


\section{Conclusion}

Flight testing is the last stage of aircraft and missile development. Flight Testing is highly expensive and potentially very risky. Unforeseen problems can lead to damage to aircraft and loss of life, both of aircrew and people on the ground. For these reasons modern flight testing is probably one of the most safety conscious professions today. Design and implementation of the PCM/FM Telemetry Systems is very important for successful evaluation of the testing prototype of aircraft and missile. The configuration of the Flight Test Instrumentation System is based on the General Plan of Testing of any new aircraft (or significant improvement to an existing aircraft) and missile. The PCM/FM system encompasses subsystems for airborne data acquisition and the compatible ground telemetry system. The airborne part includes a variety of component for measurement all relevant aircraft parameters with different amplitude and frequencies characteristics under unfavorable environmental condition. The ground telemetry system receive signal from flying object, performs real time data processing, test control and postprocesing data for post flight analysis. The Flight Test Instrumentation System has open architecture includes computer interface, high resolution and good signal to noise ratio.

\section{References}

[1] Z.Filipovic., "Contribution of synthesis PCM/FM Telemetry System for aircraft and missile testing ”, Doctors Thesis , Military Academy, Belgrade, Serbia, 2005.

[2] Range Commanders Council Telemetry Group, "Engineering Handbook", 2005.

[3] Borek,A. Pool, "Basic Principles Of Flight Test Instrumentation Engineering", AGARD Dograph No.160,Vol.1.1994.

[4] Paul D. K.,"Moder Telemetry Processing and Display System", Proceedings of the International Telemetering Conference, CD-ROM/ITC/USA '83-'99.

[5] Chuck D.David P., "A missile Instrumentation System Design Approach”, Proceedings of the International Telemetering Conference,CD- ROM/ITC/USA ,'83-'99.

[6] C.Fewer , S. Wilmot , "Enhancing The PCM/FM Link", ", Proceedings of the International Telemetering Conference, CD-ROM/ITC/USA '2009.

[7] Gary G. J., “Advaced Data Acquistion and Processing Systems(ADAPS)”, Proceedings International Telemetering Conference, CD-ROM/ITC/US, '83-'99.

[8] Michael F. L., "Air Force Flight Test Instrumentation System", Proceedings of the of the International Telemetering Conference, CD-ROM/ITC/USA, '83-'99.

[9] Range Commanders Council Telemetry Group, "IRIG Standard 106-04”, May 2004. 